\title{
CHARACTERIZATION OF NEW EXCIMER PUMPED UV LASER DYES 3. p-Quinqui-, Sexi-, Octi- and Deciphenyls
}

\author{
C. J. SELISKAR* and D. A. LANDIS \\ Chemistry Department, University of Cincinnati, Cincinnati, OH 45221-0172
}

J. M. KAUFFMAN and M. A. AZIZ

Chemistry Department, Philadelphia College of Pharmacy and Science, 600 South 43rd St., Philadelphia, PA 19104-4495

\section{R. N. STEPPEL}

Exciton, Inc., P.O. Box 31126, Overlook Station, Dayton, OH 45431

\section{J. KELLEY, YUANXI QIN and A. GHIORGHIS}

Chemistry Department, Massachusetts College of Pharmacy and Allied Health Sciences, 179 Longwood Ave., Boston, MA 02115

(Received 9 April 1992; in final form 15 May 1992)

\begin{abstract}
The spectroscopic and lasing characteristics of a series of $308 \mathrm{~nm}$ pumped p-oligophenylenes with five or more phenyl rings have been studied. Quantitative comparisons of the absorption spectra of these dyes with numerous other dyes in this class of compounds can be made by defining a parameter, theta, as the sum of the number of phenyl rings and the number of ring-bridges. Statistically good linear regressions result from the correlations of molar extinction with theta and from wavelength of absorbance with the reciprocal of theta. We find that these correlations provide good predictive information in deciding which dyes to attempt to synthesize for testing. The lasing characteristics of the dyes studied show that these dyes are amongst the best dyes in this class of dyes. Some of these dyes have exceptionally long lifetimes coupled with acceptable conversion efficiencies.
\end{abstract}

KEY WORDS : p-Oligophenylene, laser dye, excimer pumped, molar extinction coefficient, absorption, fluorescence.

\section{INTRODUCTION}

The oligophenylenes have proven to be good laser dyes in the near ultraviolet region. ${ }^{1}$ In the case of $308 \mathrm{~nm}$ excimer pumping, many dyes in this class of molecules are attractive since their first singlet-singlet absorptions are strong and typically in this wavelength region, providing for efficient optical pumping. In the first two papers in this series, ${ }^{2,3}$ we presented our work on the $308 \mathrm{~nm}$ excimer laser pumping of new derivatives of p-terphenyl and p-quaterphenyl. In this paper we extend our results to several new oligophenylenes with five or more phenyl units.

\footnotetext{
* To whom correspondence is to be sent.
} 


\section{EXPERIMENTAL DETAILS}

The experimental apparatus and methods used in this study have been described in detail previously. ${ }^{2-4}$ Dyes discussed in this paper have been examined over a period of months as they have become available. Dye lifetime and conversion efficiency data are obtained from measurements done under identical operating conditions. In particular, before each dye study the laser system was carefully tuned for optimal performance. In addition, the tuning was also periodically checked by the insertion of a standard dye (BBQ, Exciton, Inc., Dayton, $\mathrm{OH}$ ) to gauge performance. In this manner, results obtained months apart, especially for dye lifetimes, could be related quantitatively. On the other hand, it must be recognized that the measurement of dye lifetimes is sensitive to many experimental variables and the resultant lifetime values are probably only reliable to within $20 \%$.

In this study $120 \mathrm{ml}$ volumes were used in the small Lambda Physik circulators with no cover gas circulation. The optimum dye concentrations listed in Table 2 are for the oscillator-preamplifier stage of the dye laser. The amplifier concentrations were one-third of the listed values.

The dyes described in this study were synthesized by means of the general methods described in previous works. ${ }^{5-7}$ Purifications included at least one chromatographic extraction from alumina and/or silica gel. Carbon and hydrogen assays were determined for most of the dyes and were in satisfactory agreement with calculations. In general, the purification of these dyes is very difficult and several of the dyes studied contained small amounts of impurities which could not be completely removed by the techniques used for purification. We clearly identify these dyes in the Tables. The solvent p-dioxane was purchased from Fisher Scientific Co. as a Certified ACS grade solvent, and it was used only when freshly opened.

The absorption spectra were determined in cyclohexane solution, typically at about $10^{-5} \mathrm{M}$, in $1 \mathrm{~cm}$ pathlength cells using a Shimadzu UV265 spectrophotometer. Fluorescence spectra were determined on the same solutions used for absorbance measurements by using a Perkin-Elmer LS-5B. Excitation was done at the longest wavelength absorption maximum, and the fluorescence spectra were not corrected for instrument response.

\section{RESULTS AND DISCUSSION}

The dyes studied are identified and numbered in Table 1. Pertinent performance characteristics and spectroscopic constants for these dyes are summarized in Tables 2 and 3. For the sake of discussion, we have organized the dyes based on the number of phenyl rings and the ordering of dyes within each group is by the wavelength of maximum laser intensity.

General Photophysical Properties of Dyes

\section{Absorbance spectra.}

The absorbance and fluorescence spectra of a typical dye are shown in Figure 1. The spectroscopic parameters molar extinction and transition wavelength (see, for 
Table 1 Chemical structures of laser dyes

Dye number Chemical Structure

1
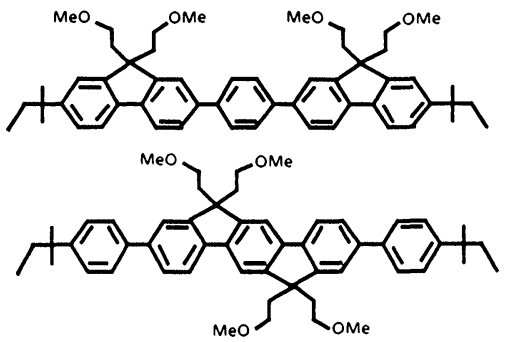

3

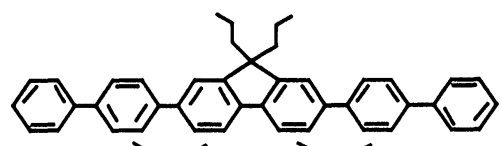

4

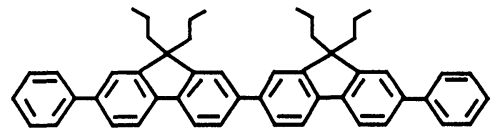

5

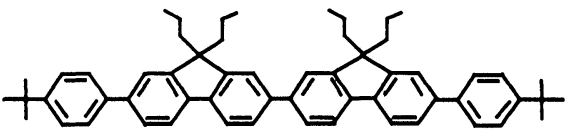

6

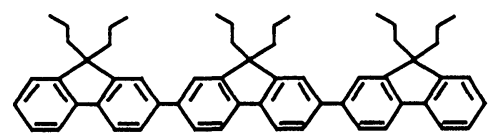

7

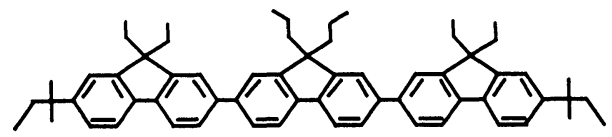

8

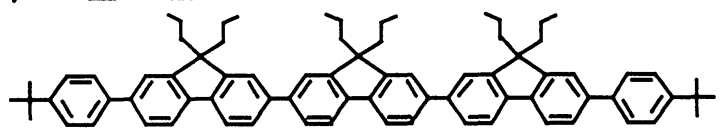

9

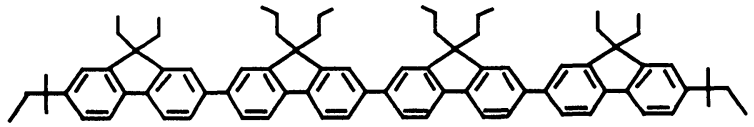

10

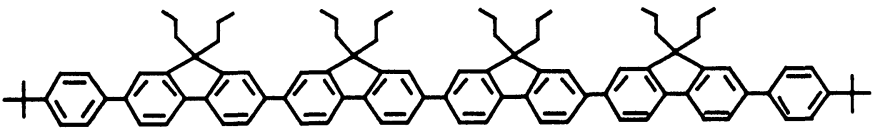

The dye structures are as follows:

1, 1,4-bis [7-t-amyl-9,9-bis (2-methoxyethyl)-2-fluorenyl]benzene ;

2, 2,8-bis (4-t-amylphenyl)-6,6,12,12-tetrakis ( 2 -methoxyethyl)indeno[ 1,2-b] fluorene:

3, 2,7-bis (4-biphenyl)-9,9-dipropylfluorene;

4, 7,7'-diphenyl-9,9,9', $9^{\prime}$-tetrapropyl-2,2'-bifluorene;

5, 7,7'-bis (4-t-amylphenyl)-9,9,9', '9'-tetrapropyl-2,2'-bifluorene ;

$6,9,9,9^{\prime}, 9^{\prime}, 9^{\prime \prime}, 9^{\prime \prime}$-hexapropyl-2,2': $7^{\prime}, 2^{\prime \prime}$-terfluorene;

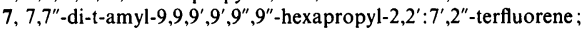

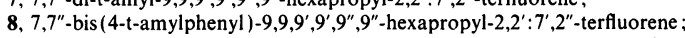

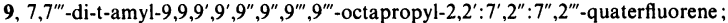

10, $7,7^{\prime \prime \prime}$-bis (4-t-amylphenyl )-9,9, $9^{\prime}, 9^{\prime}, 9^{\prime \prime}, 9^{\prime \prime}, 9^{\prime \prime \prime}, 9^{\prime \prime \prime}$-octapropyl-2, $2^{\prime}: 7^{\prime}, 2^{\prime \prime}: 7^{\prime \prime}, 2^{\prime \prime \prime}$-quaterfluorene. 
Table 2 Laser performance data for oligophenyl dyes

\begin{tabular}{|c|c|c|c|c|c|c|}
\hline \multirow[b]{2}{*}{ Dye } & \multicolumn{2}{|c|}{ Wavelength data $(\mathrm{nm})$} & \multirow[b]{2}{*}{ FWHM $^{1}$} & \multirow{2}{*}{$\begin{array}{l}\text { Conc. opt. } \\
\text { (M) }\end{array}$} & \multirow{2}{*}{$\begin{array}{l}\text { Conv. eff. } \\
(\%)\end{array}$} & \multirow{2}{*}{$\begin{array}{l}\text { Half-life } \\
\left(\mathrm{kJ} \cdot \mathrm{L}^{-1}\right)\end{array}$} \\
\hline & Maxima & Range & & & & \\
\hline & & & Quinquiphenyls & & & \\
\hline $1^{2}$ & 396,403 & $\begin{array}{l}391-412 \\
(21)\end{array}$ & $\begin{array}{l}399-409 \\
(10)\end{array}$ & $4.6 \times 10^{-4}$ & 11.3 & 800 \\
\hline $1 A^{3}$ & 385,404 & $\begin{array}{l}381-416 \\
(35)\end{array}$ & $\begin{array}{l}396-411 \\
(15)\end{array}$ & $4.8 \times 10^{-4}$ & 11.2 & 670 \\
\hline 2 & 397,408 & $\begin{array}{l}389-416 \\
(27)\end{array}$ & $\begin{array}{l}403-413 \\
(10)\end{array}$ & $8.0 \times 10^{-4}$ & 9.9 & 630 \\
\hline 3 & 394,406 & $\begin{array}{l}384-416 \\
(32)\end{array}$ & $\begin{array}{l}\text { Sexiphenyls } \\
398-413 \\
(15)\end{array}$ & $5.0 \times 10^{-4}$ & 9.8 & 480 \\
\hline $4^{2,4}$ & 400,411 & $\begin{array}{l}394-421 \\
(27)\end{array}$ & $\begin{array}{l}406-418 \\
(12)\end{array}$ & $7.3 \times 10^{-4}$ & 12.7 & 1000 \\
\hline & 400,411 & $\begin{array}{l}394-420 \\
(26)\end{array}$ & $\begin{array}{l}406-417 \\
(11)\end{array}$ & $7.3 \times 10^{-4}$ & 11.0 & 400 \\
\hline 5 & 395,414 & $\begin{array}{l}390-427 \\
(37)\end{array}$ & $\begin{array}{l}406-422 \\
(16)\end{array}$ & $2.8 \times 10^{-3}$ & 6.7 & 1100 \\
\hline 6 & 406,416 & $\begin{array}{l}400-425 \\
(25)\end{array}$ & $\begin{array}{l}410-421 \\
(11)\end{array}$ & $7.8 \times 10^{-4}$ & 10.8 & 440 \\
\hline 7 & 406,416 & $\begin{array}{l}398-430 \\
(32)\end{array}$ & $\begin{array}{l}404-426 \\
(22)\end{array}$ & $9.8 \times 10^{-4}$ & 12.0 & 800 \\
\hline 8 & 410,428 & $\begin{array}{l}408-439 \\
(31)\end{array}$ & $\begin{array}{l}\text { Octiphenyls } \\
419-436 \\
(17)\end{array}$ & $9.6 \times 10^{-4}$ & 12.5 & 870 \\
\hline 9 & 412,429 & $\begin{array}{l}408-442 \\
(34)\end{array}$ & $\begin{array}{l}409-437 \\
(28)\end{array}$ & $6.8 \times 10^{-4}$ & 9.4 & 315 \\
\hline 10 & 416,433 & $\begin{array}{l}412-442 \\
(30)\end{array}$ & $\begin{array}{l}\text { Deciphenyl } \\
427-438 \\
(11)\end{array}$ & $5.8 \times 10^{-4}$ & 9.6 & 350 \\
\hline
\end{tabular}

${ }^{1}$ Values given in parentheses are tuning curve widths in $\mathrm{nm}$.

${ }^{2}$ Shows some evidence of contamination; tuning curve shows extra minor feature (s) not attributable to dye.

${ }^{3}$ The very similar analogue of dye $\mathbf{1}$, dye $\mathbf{1 A}$, containing ethyl side-chains as opposed to methoxyethyl side-chains was made and the lasing parameters are shown here.

${ }^{4}$ This dye was prepared independently in two different batches; the second set of data listed corresponds to the preparation which showed some evidence of contamination.

example, data presented in Table 3 and also Table 1 of ref. 2) for the first singlet-singlet $\left(\mathrm{S}_{0} \rightarrow \mathrm{S}_{1}\right)$ transition of these dyes are remarkably regular. Furthermore, when viewed in the context of other dyes of this class, these spectroscopic constants are amazingly consistent with the trends established by the results of other investigators. ${ }^{8-14}$

In using our own previous experience and that of others as a guide for designing new dye structures, we have found it useful to be able to predict the wavelength and molar extinction of the $S_{0} \rightarrow S_{1}$ transition of a hypothetical dye structure. On the basis of such predictions, we then select promising dye structures to synthesize. In this process, we define a quantity theta by

$$
\text { theta }=\text { \# phenyl-rings }+ \text { \# hydrocarbon ring-bridges. }
$$

In our predictive analysis we have considered only a homologous series of p-oligophenylenes with 1- or 2-carbon bridges, with and without terminal t-alkylation. 
Table 3 Absorption and fluorescence data for oligophenyl dyes

\begin{tabular}{|c|c|c|c|c|}
\hline Dye & $\begin{array}{l}\text { Absorbance } \\
\text { maxima } \\
(\mathrm{nm})\end{array}$ & Molar extinction & Fluorescence maxima $(\mathrm{nm})$ & $\begin{array}{l}\text { Stokes shift } \\
(\mathrm{nm})\end{array}$ \\
\hline \multicolumn{5}{|c|}{ Quinquiphenyls } \\
\hline 1 & 332 & 76,000 & $380,399,421(\mathrm{sh})$ & 48 \\
\hline $\mathbf{1 A}$ & 332 & 76,000 & $381,400,420(\mathrm{sh})$ & 49 \\
\hline 2 & 350 & 75,000 & $383,401,423(\mathrm{sh})$ & \\
\hline & 366 & 70,000 & & 17 \\
\hline \multicolumn{5}{|c|}{ Sexiphenyls } \\
\hline 4 & 347 & 86,000 & $387,408,430(\mathrm{sh})$ & 40 \\
\hline 5 & 346 & 77,000 & $389,409,435(\mathrm{sh})$ & 43 \\
\hline 7 & 353 & 93,000 & $397,415,440(\mathrm{sh})$ & 44 \\
\hline \multicolumn{5}{|c|}{ Octiphenyls } \\
\hline $\mathbf{8 X}$ & 357 & 98,000 & $401,423,448(\mathrm{sh})$ & 44 \\
\hline 8 & 360 & $117,000^{1}$ & $401,424,444(\mathrm{sh})$ & 41 \\
\hline 9 & 363 & 141,000 & $405,425,452(\mathrm{sh})$ & 42 \\
\hline \multicolumn{5}{|c|}{ Deciphenyl } \\
\hline 10 & 366 & 153,000 & $408,429,462(\mathrm{sh})$ & 42 \\
\hline
\end{tabular}

${ }^{1}$ This dye was prepared twice. The second preparation gave a molar extinction of 130,000 .

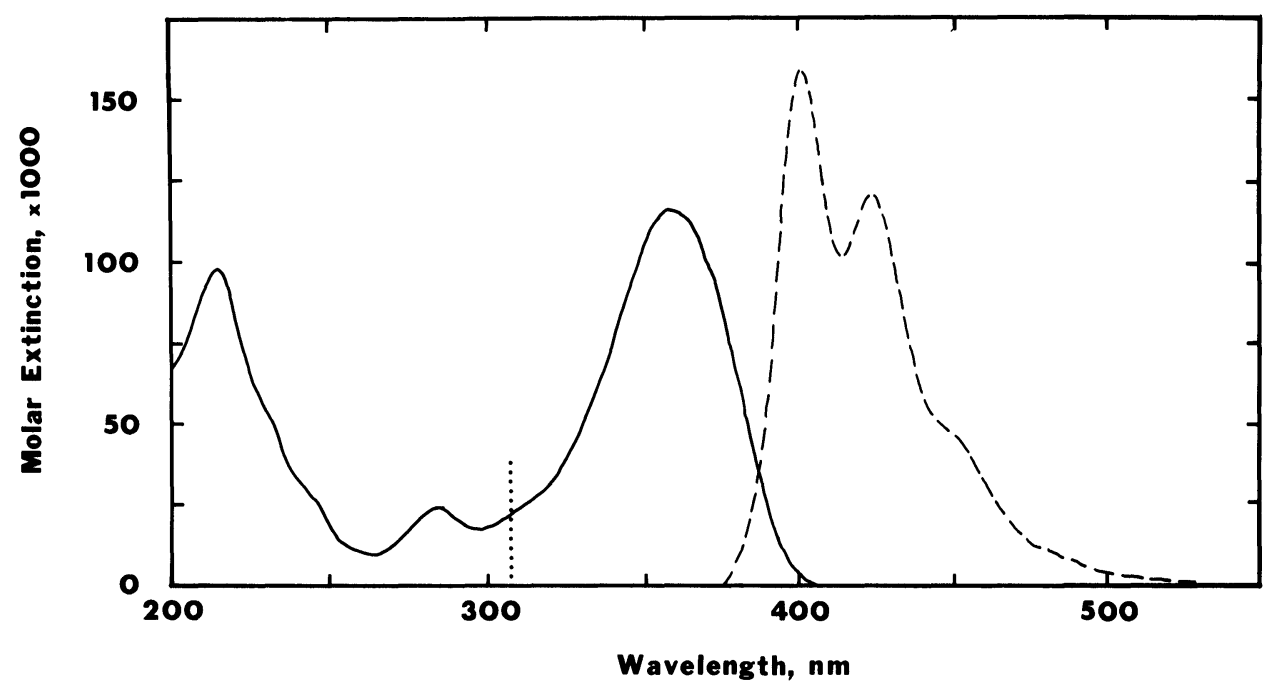

Figure 1 The molar extinction $\left(1 \cdot \mathrm{M}^{-1} \cdot \mathrm{cm}^{-1}\right)$, solid line, and the fluorescence spectrum, dashed line, of dye 8 in the solvent cyclohexane is shown. The vertical dotted line corresponds to $308 \mathrm{~nm}$ excitation.

We have used data of our own and others which were obtained in non-polar solvents, cyclohexane where possible.

As a demonstration of this predictive capacity, Figure 2 shows a plot of the molar extinction coefficient versus theta for a series of 29 p-oligophenylenes beginning with p-terphenyl. The linear regression analysis of this data set yields a value of the square of the correlation coefficient, $R$, of 0.944 . In the case of the wavelength of the 


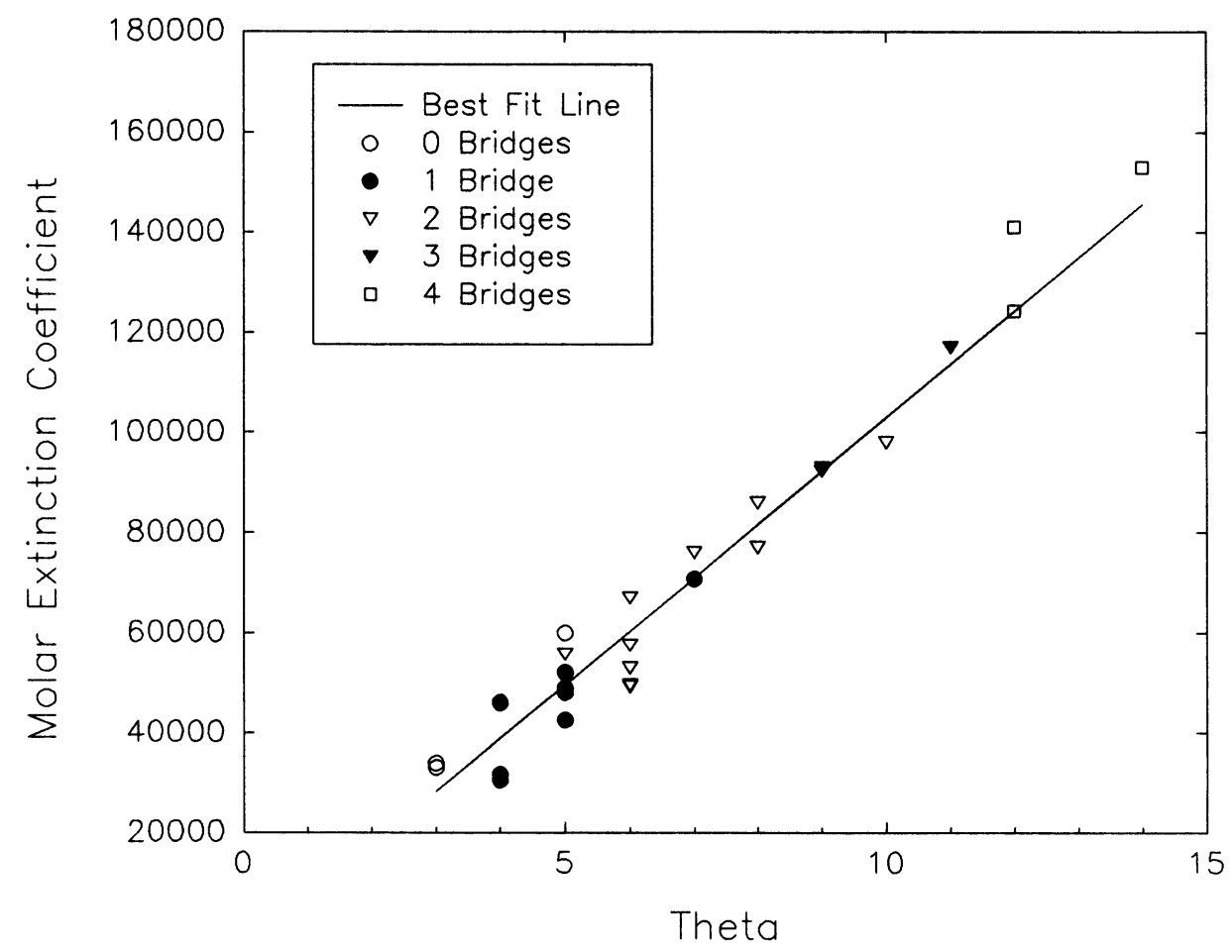

Figure 2 A plot of the molar extinction coefficient for 29 p-oligophenylenes (ring-bridged and non-bridged, with and without terminal t-alkylation dyes included ) versus the defined parameter theta is shown. The incorporated legend identifies the dyes by the number of ring bridges, thus, also identifying the number of rings for a specific value of theta.

absorbance maximum of the $S_{0} \rightarrow S_{1}$ transition in this same series of dyes, the linear correlation of the wavelength with the reciprocal of theta is equally good. The statistical analysis of this correlation yields a value of $\mathrm{R}^{2}=0.922$, with a slope of $-329.4 \mathrm{~nm} \cdot$ theta and an intercept of $386.4 \mathrm{~nm}$.

While the correlations for molar extinction and absorbance wavelength are surprisingly good and certainty useful in designing new p-oligophenylenes, we do not believe that the demonstrated linearity is rooted in some simple fundamental aspect of the spectroscopy of these molecules. Nonetheless, there are several reasons why the molar extinction and transition wavelength should be generally correlated with the parameter theta. First, as Wirth and co-workers have noted, ${ }^{9}$ the general correlation of both the molar extinction and the absorbance wavelength with the number of phenyls in the simple p-oligophenyls is very good. Our analysis quantifies this correlation for 29 bridged and non-bridged p-oligophenyls. Second, in the case of the influence of hydrocarbon ring-bridging, the bridged aromatic rings of the dyes are constrained to be planar or near planar depending on the size of the bridging structure (5- or 6-membered rings). This constraint can be expected to increase the conjugation between rings and thus generally shift the transition wavelength to longer values and enhance the associated extinction. Third, that the transition wavelength is linearly related to the reciprocal of theta, means that the transition energy, which 


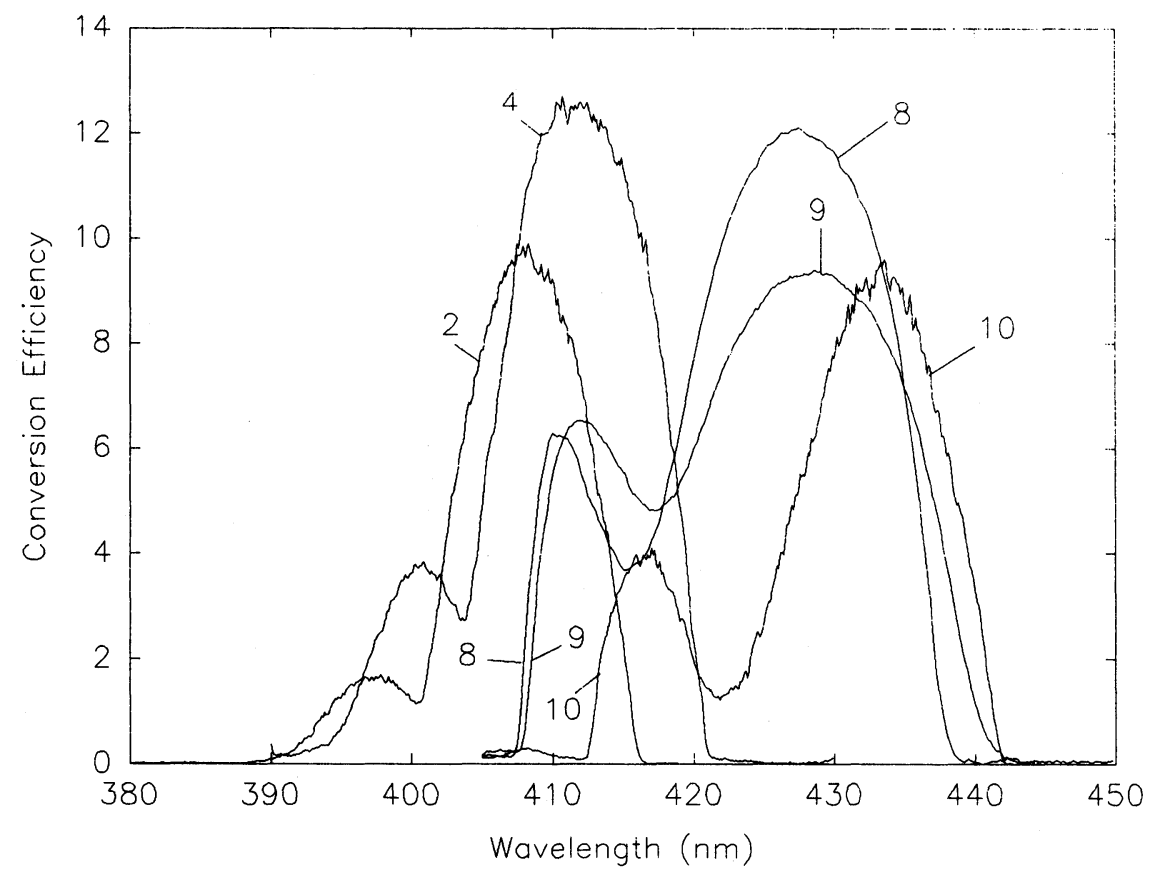

Figure 3 The tuning curves (percent conversion efficiency versus wavelength) for representative dyes in p-dioxane solvent are shown. Each tuning curve is identified by the appropriate dye number as given in Table 1.

is directly proportional to the reciprocal of the associated wavelength, is inversely related to the conjugation length.

The highest molar extinction coefficient for $\mathrm{S}_{0} \rightarrow \mathrm{S}_{1}$ of the p-oligophenylenes has been estimated by Berlman ${ }^{10}$ to be 90,000 using two different methods. The corresponding value for our sexiphenyl $7(93,000)$ exceeds that value as do those of octiphenyls 8 and 9 and deciphenyl 10 (Table 3). Thus, it appears that Berlman's estimation of the limiting values for oligophenyls have been surpassed even at the level of p-sexiphenyl. It is also now clear that the longest absorption wavelength possible, proposed by Berlman ${ }^{12}$ to be $328 \mathrm{~nm}$, and by Wirth et al. ${ }^{9}$ to be $345 \mathrm{~nm}$, has also been surpassed at the level of sexiphenyl 7, To the extent that our correlation of the reciprocal of theta with this wavelength is validly extrapolated, the present analysis predicts a limiting value of $386.4 \mathrm{~nm}$ (regression analysis intercept value), a large bathochromic shift.

\section{Fluorescence and lasing properties}

A composite of the tuning curves for selected dyes is shown in Figure 3. Some general conclusions follow on inspection of these spectra. While the absorbance spectra of the dyes are typically vibrationally unstructured, the fluorescence spectra are all vibrationally structured. This vibrational structure in the fluorescence undoubtedly has its origins in the ring vibration modes of the basic phenyl unit. The laser tuning curves which result are a product of the fluorescence curve (in p-dioxane) and the 
laser cavity efficiency curve. Thus, each tuning curve is double-featured in its appearance and consists of contributions from the first two vibronic components of the fluorescence.

On the whole, the conversion efficiencies with $308 \mathrm{~nm}$ pumping listed in Table 2 vary from 6.7 to $12.7 \%$ making any one of these dyes quite usable. However, when compared with previously measured dyes as the p-quaterphenyls, ${ }^{3}$ these conversion efficiencies are generally somewhat lower. This might be attributable to the fact that in this series of dyes $308 \mathrm{~nm}$ excitation pumps these molecules to levels above the first excited singlet state where possibly there are more decay channels available for energy dissipation than just internal conversion to $S_{1}$ and subsequent fluorescence.

Compared with the commercially available dye BBQ, which we use as a convenient and familiar reference dye (see refs. 2-4), all of the dye lifetimes in Table 2 are very much longer. Thus, even the shortest-lived of these new dyes represents a large improvement in stability. Our previous finding that incorporation of $\mathrm{t}$-alkyl groups as auxofluors conferred the greatest stability upon $308 \mathrm{~nm}$ pumped p-terphenyl and p-quaterphenyl laser dyes was not carried over into the sexiphenyls. Overall, these new dyes represent the longest wavelength lasing dyes of the p-oligophenylenes. Because of the wavelength of the $S_{0} \rightarrow S_{1}$ transition, it is also clear that these dyes are excellent candidates for pumping with tripled Nd:YAG lasers.

Inspection of Table 2 also shows that several of the dye lifetimes are relatively low for this series of closely related dyes. In this regard, we found evidence that for this series of larger p-oligophenylenes the dye lifetime may be sensitive to slight amounts of impurities in the dye sample. We have not encountered this same sensitivity in our prior work on the smaller p-oligophenylenes. ${ }^{2-4}$ This sensitivity could be due to two inter-related factors, the coincidence of which could produce the sensitivity observed. First, given the higher degree of difficulty encountered in purifying these dyes, the preparations of these materials could be less pure than previous preparations. Second, in the case of these larger dyes, the larger synthetic intermediates necessary to the syntheses of them can much more efficiently absorb and dissipate $308 \mathrm{~nm}$ excitation, thus reducing pump radiation to the dye itself. In turn, this effect could also possibly increase the apparent lifetime of a given dye sample.

\section{Quinquiphenyls}

The quinquiphenyls 1 and 2 studied represent rationally designed structures with identical t-alkyl groups as auxoflors and identical side chains (2-methoxyethyl, which were effective in the dyes discussed in Fleitz et al. ${ }^{3}$ ). Both dyes are good dyes from the standpoint of conversion efficiency and lifetime. However, dye $\mathbf{1}$ is significantly more efficient and stable. Even with an apparent minor lasing contaminant, dye 1 has a very long lifetime and ranks as one of the better p-oligophenylenes. The data in Table 2 shows that dye $\mathbf{2}$ lases slightly to the red of dye $\mathbf{1}$ and this is attributable to the constrained planar 3-ring central structure within dye 2. Replacement of the 2-methoxyethyl side chains in dye 1, see dye 1A, did not alter the conversion efficiency, but lowered the lifetime about $16 \%$. This effect was in the same direction as we observed in the p-quaterphenyls. ${ }^{3}$ The FWHM (or the corresponding tuning ranges) of the tuning curves for these p-quinquiphenyls are typical of the p-oligophenylenes in this wavelength region, about $10 \mathrm{~nm}$. 


\section{Sexiphenyls}

Two of the p-sexiphenyls, 4 and 7, examined are excellent laser dyes and rank amongst the best we have ever studied. Both dyes $\mathbf{4}$ and $\mathbf{7}$ have excellent stability, conversion efficiency and tuning ranges. Dye 7 is exceptional in that both vibronic components of the fluorescence carry high optical gain and this is reflected in the very wide FWHM of this dye. It is interesting that the differences in ring-bridging between these two dyes (two versus three bridges) makes little difference in the other lasing properties. In other cases we have studied (refs. 2, 3 and vide infra), the number of ring-bridges makes significant differences in these properties.

Dye 5, the t-alkylated analogue of dye 4, has an excellent lifetime, but relatively poor conversion efficiency. The addition of flexible t-alkyl groups (dye 5) has broadened the tuning curve relative to dye 4, so that the FWHM of dye 5 is more desirable. This tuning curve broadening with the addition of $t$-alkyl groups is carried over in the relationship of dyes 6 and 7. In the case of dye 7, the conversion efficiency and lifetime are improved as well.

\section{Octiphenyls}

The two p-octiphenyls 8 and 9 examined differ mainly in the number and positioning of their ring-bridges. Both dyes contain t-alkyl auxoflors, a synthetic expedient. Dye $\mathbf{8 X}$, a 2-bridged analogue of $\mathbf{8}$, had a low extinction coefficient (Table 3 ), and was too insoluble to be examined for lasing properties. It is remarkable that the lasing parameters of the octiphenyl dyes differ by as much as they do. Dye $\mathbf{8}$ is one of the better oligophenylenes we have studied. Its relatively high conversion efficiency and long lifetime make it a very useful dye in the area of $428 \mathrm{~nm}$. In comparison, dye 9 is much less stable and significantly less efficient. On the other hand, inspection of Figure 3 shows that the tuning curve for the latter dye is significantly broader due to the first vibronic component lasing more efficiently. So in spite of the fact that dye 9 is not as generally good in comparison, the tuning curve is very desirable. A 4-ring-bridged analogue of dye 9 without terminal t-alkyl groups was prepared. However, this compound lacked sufficient solubility in p-dioxane to be used as a $308 \mathrm{~nm}$ pumped laser dye.

\section{Deciphenyl}

We have studied only one p-deciphenyl to date. Dye $\mathbf{1 0}$ apparently represents the longest wavelength lasing oligophenylene dye known. Its chemical structure incorporates the stability enhancements of replacement of hydrogens on the ring-bridging carbon atoms by alkylation and use of t-alkyl groups as auxofluors. However, looking at the lasing properties of this dye in p-dioxane given in Table 2, one can see that this dye is less efficient in comparison to most of the other dyes. Its lifetime value is also significantly reduced.

\section{CONCLUSIONS}

The conclusions of this work are as follows. First, the molar extinction coefficient and the absorbance wavelength of the $S_{0} \rightarrow S_{1}$ transition for a homologous series of nearly thirty p-oligophenylenes can be predicted by defining a useful parameter, theta, 
as the sum of the number of phenyl rings and the number of hydrocarbon ring-bridges. This linear correlation may be extendable to other homologous series of laser dyes as well. Second, the previously predicted limits of the molar extinction coefficient as well as the wavelength of the $S_{0} \rightarrow S_{1}$ transition for oligophenylenes has been surpassed at the level of the sexiphenyls. Third, the series of dyes examined represent a very significant improvement for the region 390-440 nm. As such they should be very useful for both $308 \mathrm{~nm}$ excimer and tripled Nd:YAG pumping sources. Fourth, the conversion efficiencies shown are slightly lower, in general, than we have found for smaller p-oligophenylenes in our previous studies. The lower observed efficiencies may be related to triplet state absorption in the lasing wavelength range, as recently reported ${ }^{15}$ for a series of unrelated laser dyes. Nonetheless, the conversion efficiencies found are reasonable $(7-13 \%)$ and are offset by the greatly increased stabilities and wavelength coverage found in this series of dyes.

\section{Acknowledgements}

This work was supported in part by a grant to CJS and RNS from the State of Ohio Thomas Edison Fund and by the State of Ohio's Academic and Research Challenge Awards to the Chemistry Department of the University of Cincinnati. Dyes 1,8 and 9 were originally prepared with the financial support from a subcontract to JMK from Purdue University based on a contract from the Texas National Research Laboratory Commission. J. A. Novinski carried out the final step in the synthesis of dye 2. A key suggestion for the preparation of the indeno [1,2-b] fluorene intermediate for dye $\mathbf{2}$ was made by R. K. Hill. Discussions with Theodore G. Pavlopoulos were very valuable. The authors thank Elaine M. Seliskar and Kathy Orchin for technical assistance in preparing the manuscript.

\section{References}

1. R. N. Steppel, "Organic Dye Lasers", in Handbook of Laser Science and Technology, Supplement 1: Lasers, edited by M. J. Weber, pp. 219-317. Boca Raton: CRC Press (1991).

2. D. J. Schneider, D. A. Landis, P. A. Fleitz, C. J. Seliskar, J. M. Kauffman and R. N. Steppel. Laser Chem., 11, 49 (1991).

3. P. A. Fleitz, C. J. Seliskar, R. N. Steppel, J. M. Kauffman, C. J. Kelley and A. Ghiorghis, Laser Chem., 11, 99 (1991).

4. D. A. Landis, D. J. Schneider and C. J. Seliskar, Laser Chem., 11, 63 (1991).

5. J. M. Kauffman, C. J. Kelley, A. Ghiorghis, E. Neister, L. Armstrong and P. Prause, Laser Chem., 7, 343 (1987).

6. J. M. Kauffman, C. J. Kelley, A. Ghiorghis, E. Neister and L. Armstrong, Laser Chem., 8, 335 (1988).

7. J. M. Kauffman, C. J. Kelley, A. Ghiorghis, E. Neister, C. J. Seliskar and R. N. Steppel, Proc. Intl. Conf. on Lasers '89, STS Press, McLean, VA (1990), p. $420 \mathrm{ff}$.

8. M. Rinke, H. Gusten and H. J. Ache, J. Phys. Chem., 90, 2661 (1986). M. Rinke, H. Gusten and H. J. Ache, J. Phys. Chem., 90, 2666 (1986).

9. H. O. Wirth, F. U. Herrmann, G. Herrmann and W. Kern, Molec. Cryst., 4, 321 (1968).

10. I. B. Berlman, Molec. Cryst., 4, 157 (1968).

11. I. B. Berlman, H. O. Wirth and O. J. Steingraber, J. Phys. Chem., 75, 318 (1971).

12. I. B. Berlman, Handbook of Fluorescence Spectra of Aromatic Molecules. New York: Academic Press (1971).

13. I. B. Berlman, J. Chem. Phys., 52, 5616 (1970).

14. U. Brackmann, Lambdachrome Laser Dyes, Lambda Physik, D-3400 Gottingen, West Germany (1986).

15. T. G. Pavlopoulos and D. J. Golich, J. Appl. Phys., 67, 1203 (1990). 